J. Appl. Numer. Optim. 1 (2019), No. 2, pp. 117-129

Available online at http://jano.biemdas.com

https://doi.org/10.23952/jano.1.2019.2.03

\title{
A HENIG CONICAL REGULARIZATION APPROACH FOR CIRCUMVENTING THE SLATER CONUNDRUM IN LINEARLY $\ell_{+}^{p}$-CONSTRAINED LEAST SQUARES PROBLEMS
}

\author{
LIDIA HUERGA ${ }^{1}$, AKHTAR A. KHAN ${ }^{2}$, MIGUEL SAMA $^{1, *}$ \\ ${ }^{1}$ Departamento de Matemática Aplicada, Universidad Nacional de Educación a Distancia, \\ Calle Juan del Rosal, 12, 28040 Madrid, Spain \\ ${ }^{2}$ Center for Applied and Computational Mathematics, School of Mathematical Sciences,
} Rochester Institute of Technology, 85 Lomb Memorial Drive, Rochester, New York, 14623, USA

\begin{abstract}
In this paper, we study a linearly $\ell_{+}^{p}$-constrained least-squares problem. We develop the Henig conical regularization approach as a unified framework to deal with the lack of Slater-type constraint qualification. We establish some stability estimates for the regularized problems. For the separable case, $p \in[1, \infty)$, we provide an explicit characterization of the Henig dilating cones associated with $\ell_{+}^{p}$ and the associated regularized KKT systems. For the non-separable case $\ell_{+}^{\infty}$, we give a condition which ensures that the solution of the least-squares problem can be approximated by regularized solutions whose dual solutions do not contain any finitely additive singular part.
\end{abstract}

Keywords. Perturbation theory; Convex optimization; $\ell^{p}$ spaces; Dilating cones.

2010 Mathematics Subject Classification. 35R30, 49N45, 65J20, 65J22, 65M30.

\section{INTRODUCTION}

Let $V$ and $X$ be real Hilbert spaces with $\|\cdot\|_{V}$ and $\|\cdot\|_{X}$ as their norms. Given an exponent $p \in[1, \infty]$, we consider the classical sequence space $\ell^{p}$ equipped with its natural ordering $\leq_{\ell_{+}^{p}}$. That is, for any $\mathbf{y}=\left\{y_{i}\right\}_{i \in \mathbb{N}} \in \ell^{p}$ and $\mathbf{z}=\left\{z_{i}\right\}_{i \in \mathbb{N}} \in \ell^{p}$, we have $\mathbf{y} \leq_{\ell_{+}^{p}} \mathbf{z}$, if and only if, $y_{i} \leq z_{i}$, for every $i \in \mathbb{N}$, where $\mathbb{N}$ is the set of positive integers.

Consider the following linearly $\ell^{p}$-constrained least-squares optimization problem:

$$
\text { Minimize } J(x):=\frac{1}{2}\left\|S x-v_{d}\right\|_{V}^{2}+\frac{\kappa}{2}\left\|x-x_{d}\right\|_{X}^{2} \text { subject to } G x \leq_{\ell_{+}^{p}} \mathbf{w}, x \in X . \quad\left(Q_{p}\right)
$$

Here $S: X \rightarrow V$ and $G: X \rightarrow \ell^{p}$ are linear and bounded operators, $\kappa>0$ is a given parameter, and $x_{d} \in X$, $v_{d} \in V$, and $\mathbf{w} \in \ell^{p}$ are the given elements.

Optimization problem $\left(Q_{p}\right)$, which evidently has the unique solution $\bar{x}_{p}$, is a constrained optimization problem with countably infinite number of linear constraints. Such problems commonly appear in many important models, for example, in the so-called infinite horizon or infinite network problems, see [4, $5,7,24,26]$. The main obstacle in an adequate treatment of infinite-dimensional problems with linear constraints is the existence of the dual solutions. In strict contrast to finite-dimensional optimization problems, in infinite-dimensional optimization problems, the existence of dual solutions imposes severe

\footnotetext{
${ }^{*}$ Corresponding author.
}

E-mail addresses: lhuerga@ind.uned.es (L. Huerga), aaksma@ rit.edu (A.A. Khan), msama@ind.uned.es (M. Sama).

Received July 23, 2019; Accepted August 14, 2019. 
conditions on the constraint space such as the existence of the interior points for the ordering cones, see $[2,4]$. Note that, if we choose $p=\infty$, then the ordering cone $\ell_{+}^{\infty}$ has nonempty interior, and the Slater constraint qualification holds, that is, there exists an element $\tilde{x} \in X$ such that

$$
G \tilde{x}-\mathbf{w} \in-\operatorname{int}\left(\ell_{+}^{\infty}\right),
$$

and, consequently, there exists a solution $\mu^{*} \in\left(\ell_{+}^{\infty}\right)^{*}$ of the dual problem associated to $\left(Q_{\infty}\right)$. Moreover, the dual solution $\mu^{*}$, along the primal solution $\bar{x}_{\infty}$, verify the corresponding KKT optimality conditions

$$
\begin{aligned}
D J\left(\bar{x}_{\infty}\right)+\mu^{*} \circ G & =0, \\
\mu^{*}\left(G \bar{x}_{\infty}-\mathbf{w}\right) & =0, \\
G \bar{x}_{\infty}-\mathbf{w} & \leq \ell_{+}^{\infty} 0 .
\end{aligned}
$$

Even in this seemingly normal situation, there are complications. Firstly, $\ell^{\infty}$ is not separable, and the main drawback is that the dual elements $\mu^{*}$ may have a finitely additive (singular) part which makes its numerical computation challenging or even impossible (see [22]). In applications, for example in the economic models, the multipliers represent prices, whereas an interpretation of the singular part remains unclear (see [21, 23]). On the other hand, for $p<\infty$, we have $\operatorname{int}\left(\ell_{+}^{p}\right)=\emptyset$ and hence (1.1) is meaningless. Although one can resort to some other constraint qualifications, the dual problem is simply unsolvable in most cases, as we see in Example 1. A similar situation arises in PDE constrained optimization problems where the constraint space is $L^{P}$, the space of Lebesgue integrable functions, see [17]. This problem, with a possible singular multiplier in $p=\infty$, and non-existence of interior points in the case $p \in[1, \infty)$, has recently been named as the Slater conundrum in the literature, $[4,20]$. In this context, in this paper, we exploit a general technique, dubbed the conical regularization, which was devised in [15] to circumvent the difficulties associated with the failure of the Slate-type constraint qualifications. For $\left(Q_{p}\right)$, the conical regularization consists of replacing the ordering cone by a family of dilating cones. Conical regularization scheme has been tested by using different families of dilating cones [10, 11, 12], it has been applied to different PDE constrained optimization problems, see [14, 15, 18], and extended to multiobjective and vector optimization problems, see [13, 25].

This paper aims to conduct a thorough theoretical and numerical investigation of $\left(Q_{p}\right)$ by using the Henig conical regularization, that is, the conical regularization which is based on Henig dilating cones (see [3, 8]). Given $\ell_{+}^{p}$, let $\left\{\ell_{+, \varepsilon}^{p}\right\}$, where $\varepsilon \in(0,1)$, be an associated family of Henig dilating cones, that is, for each $\varepsilon \in(0,1), \ell_{+, \varepsilon}^{p}$ is a closed, convex, and pointed cone with a nonempty interior such that the following two conditions hold:

- $\ell_{+}^{p} \backslash\{0\} \subset \operatorname{int}\left(\ell_{+, \varepsilon}^{p}\right)$, for every $\varepsilon \in(0,1)$.

$$
\text { - } \ell_{+}^{p}=\bigcap_{\varepsilon>0} \ell_{+, \varepsilon}^{p} \text {. }
$$

The regularized family $\left(Q_{p}^{\varepsilon}\right)$ is then defined by replacing $\ell_{+}^{p}$ in $\left(Q_{p}\right)$ by $\ell_{+, \varepsilon}^{p}$ as follows

$$
\text { Minimize } J(x):=\frac{1}{2}\left\|S x-v_{d}\right\|_{V}^{2}+\frac{\kappa}{2}\left\|x-x_{d}\right\|_{X}^{2} \text { subject to } G x \leq_{\ell_{+, \varepsilon}^{p}} \mathbf{w}, x \in X .
$$

For each $\varepsilon>0$, the regularized problem $\left(Q_{p}^{\varepsilon}\right)$ has a unique solution $x_{p}^{\varepsilon}$. Furthermore, each regularized solution $x_{p}^{\varepsilon}$ can be solved through KKT optimality conditions, and $x_{p}^{\varepsilon} \rightarrow \bar{x}_{p}$ when $\varepsilon \rightarrow 0$. In this work, we develop the Henig conical regularization scheme for problems $\left(Q_{p}\right)$. We distinguish between the two 
main cases, namely, the case when $p \in[1, \infty)$ and the case when $p=\infty$. We prove an explicit characterization of the dual of Henig cones $\ell_{+, \varepsilon}^{p}$, when $p<\infty$ (Proposition 2.2). This result permits us to derive an explicit characterization of regularized KKT systems (Proposition 2.3). In the main result of the paper, by using some recent estimates for the regularization error established in [12], we give conditions such that the solution $\bar{x}_{\infty}$ can be approximated by regularized solutions whose dual solutions do not contain any finitely additive part (Theorem 2.8). We then improve the convergence rate to $\left\|x_{p}^{\varepsilon}-\bar{x}_{p}\right\|_{X}=\mathscr{O}(\varepsilon)$ under an additional assumption on the trajectories of the regularized solutions. Under this assumption, for $p \in(1, \infty)$, we prove an equivalence among the norm boundedness of the regularized multipliers, the linear convergence rate, and the regularity of problem $\left(Q_{p}\right)$. We also provide two numerical examples to illustrate our theoretical results.

1.1. Notations and Preliminaries. We now briefly describe the notations that will be used in this paper. For a generic Banach space $Z$, we denote its dual by $Z^{*}$ and its norm by $\|\cdot\|_{Z}$. The strong and the weak convergence in any space will be represented by $\rightarrow$ and $\rightarrow$ respectively. By $\ell^{p}$ we denote the Banach space of all sequences $\mathbf{y}=\left\{y_{i}\right\}_{i \in \mathbb{N}}$ endowed with the norm

$$
\|\mathbf{y}\|_{p}:=\|\mathbf{y}\|_{\ell^{p}}=\left(\sum_{i=1}^{\infty}\left|y_{i}\right|^{p}\right)^{\frac{1}{p}}<\infty,
$$

for $p \in[1, \infty)$, and

$$
\|\mathbf{y}\|_{\infty}:=\|\mathbf{y}\|_{\ell^{\infty}}=\max \left\{\left|y_{i}\right|: i \in \mathbb{N}\right\}<\infty,
$$

for $p=\infty$. By $\mathbf{e}_{j}=\left\{e_{i}^{j}\right\}_{i \in \mathbb{N}} \in \ell^{p}$, we denote the element of the canonical basis of $\ell^{p}$ verifying $e_{i}^{j}=1$ for $i=j, e_{i}^{j}=0$ for $i \neq j$. By $\left\{e_{i}^{*}\right\} \subset\left(\ell^{p}\right)^{*}$, we denote the associated biorthogonal functionals satisfying $e_{i}^{*}\left(\mathbf{e}_{j}\right)=1$ for $i=j, e_{i}^{*}\left(\mathbf{e}_{j}\right)=0$ for $i \neq j$. We consider the natural ordering cone $D=\ell_{+}^{p}$ given by the positive coordinates

$$
\ell_{+}^{p}=\left\{\mathbf{y} \in \ell^{p}: y_{i} \geq 0 \text { for every } i \in \mathbb{N}\right\} .
$$

For $p \in[1, \infty)$, every dual element $\mu_{p} \in\left(\ell_{+}^{p}\right)^{*}=\left\{\mu \in\left(\ell^{p}\right)^{*}: \mu(\mathbf{d}) \geq 0, \forall \mathbf{d} \in \ell_{+}^{p}\right\}$ can be identified with a sequence $\mu_{p} \equiv\left\{\mu_{i}^{p}\right\}_{i \in \mathbb{N}} \in \ell^{q}$, where $q$ is the conjugate exponent satisfying $p^{-1}+q^{-1}=1$. In other words, $\mu_{p}(\mathbf{d})=\sum_{i=1}^{\infty} \mu_{i}^{p} d_{i}$. For $p=\infty$, every $\mu_{\infty} \in\left(\ell_{+}^{\infty}\right)^{*}$ can be expressed as the sum of a countably additive part $\left\{\mu_{i}^{\infty}\right\}_{i \in \mathbb{N}} \in \ell_{+}^{1}$ and a purely finitely additive part $\mu_{\text {sing }} \in\left(\ell^{\infty}\right)^{*}$, that is,

$$
\mu_{\infty}(\mathbf{d})=\sum_{i=1}^{\infty} \mu_{i}^{\infty} d_{i}+\mu_{\text {sing }}(\mathbf{d}) .
$$

For more more details, see, for example, [1,22].

We begin with a formal definition of the Henig dilating cone $\ell_{+, \varepsilon}^{p}$, initially proposed by Borwein and Zhuang [3] for the infinite-dimensional setting, extending the notion given in finite-dimensional by Henig [9].

Let $\beta=\left\{\beta_{i}\right\}_{i \in \mathbb{N}} \in \ell^{1}$ be a fixed sequence with strictly positive coordinates $\beta_{i}>0$. The associated linear functional $\beta(\mathbf{x})=\sum_{i=1}^{\infty} \beta_{i} x_{i}$ belongs to any dual $\left(\ell^{p}\right)^{*}$ for each $p \in[1, \infty]$. In fact,

$$
\beta \in\left(\ell_{+}^{p}\right)^{\natural}=\left\{\mu \in\left(\ell^{p}\right)^{*}: \mu(\mathbf{d})>0, \forall \mathbf{d} \in \ell_{+}^{p} \backslash\{\mathbf{0}\}\right\}
$$

is a strictly positive functional. For each $p \in[1, \infty]$, we consider the normalized element

$$
\beta_{p}=\left\{\beta_{i}^{p}\right\}_{i \in \mathbb{N}}:=\|\beta\|_{q}^{-1} \beta \text {. }
$$


By $\Theta_{p}$, we denote the associated basis of cone $\ell_{+}^{p}$ given by

$$
\Theta_{p}=\left\{\mathbf{d} \in \ell_{+}^{p}: \beta_{p}(\mathbf{d})=1\right\},
$$

see [16, Theorem 2.2.12]. Aided with these tools, for each $\varepsilon \in(0,1)$, the corresponding Henig dilating cone $\ell_{+, \varepsilon}^{p}$ is defined by the following expression

$$
\ell_{+, \varepsilon}^{p}=\operatorname{cl}\left[\operatorname{cone}\left(\Theta_{p}+\varepsilon B_{p}\right)\right],
$$

where $B_{p}=\left\{\mathbf{d} \in \ell^{p}:\|\mathbf{d}\|_{p} \leq 1\right\}$ denotes the closed unit ball of $\ell^{p}$.

\section{Henig Conical Regularization}

Throughout this section, let $p \in[1, \infty]$ be a fixed exponent. We aim to study the Henig conically regularized problems $\left(Q_{p}^{\varepsilon}\right)$. By

$$
F_{p}:=\left\{x \in X: \mathbf{G x}-\mathbf{w} \leq_{\ell_{+}^{p}} 0\right\},
$$

we denote the feasible set of problem $\left(Q_{p}\right)$, which we assume to be nonempty. Analogously, by

$$
F_{p, \varepsilon}=\left\{x \in X: \mathbf{G x}-\mathbf{w} \leq_{\ell_{+, \varepsilon}^{p}} 0\right\},
$$

we denote the feasible set of problem $\left(Q_{p}^{\varepsilon}\right)$ for each $\varepsilon \in(0,1)$. For $\varepsilon>0$, recalling that $x_{p}^{\varepsilon}$ denotes the unique solution to $\left(Q_{p}^{\varepsilon}\right)$, we set $\mathbf{q}_{p}^{\varepsilon}:=\mathbf{G} \mathbf{x}_{\mathbf{p}}^{\varepsilon}-\mathbf{w}$. Furthermore, we set $\overline{\mathbf{q}}_{p}:=\mathbf{G} \overline{\mathbf{x}}_{\mathbf{p}}-\mathbf{w}$, and assume the condition

$$
\overline{\mathbf{q}}_{p}=\mathbf{G} \overline{\mathbf{x}}_{\mathbf{p}}-\mathbf{w} \neq 0
$$

which provides a non-trivial feasible point such that the regularized optimality system is solvable (see [15]). Although the original regularization scheme given in [15, Theorems 3.1-3] is given for a Hilbert constraint space $Y$, the proofs of that results are still valid for a general Banach space. Therefore we can apply these results to the present case, and directly obtain the following:

Proposition 2.1. For each $\varepsilon \in(0,1)$, there is a unique solution $x_{p}^{\varepsilon}$ to problem $\left(Q_{p}^{\varepsilon}\right)$. Moreover, the regularized solutions $\left\{x_{p}^{\varepsilon}\right\}$ converge strongly to $\bar{x}_{p}$, as $\varepsilon \rightarrow 0$. Finally, for each $\varepsilon \in(0,1)$, there exists a

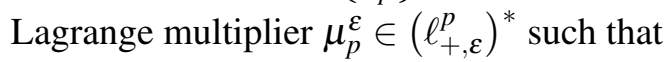

$$
\begin{aligned}
D J\left(x_{p}^{\varepsilon}\right)+\mu_{p}^{\varepsilon} \circ G & =0 \\
\boldsymbol{\mu}_{\boldsymbol{p}}^{\boldsymbol{\varepsilon}}\left(\mathbf{G} \mathbf{x}_{\mathbf{p}}^{\varepsilon}-\mathbf{w}\right) & =0 \\
\mathbf{G} \mathbf{x}_{\mathbf{p}}^{\varepsilon}-\mathbf{w} & \leq_{\ell_{+, \varepsilon}^{p}} 0 .
\end{aligned}
$$

In the following, we will distinguish two different cases.

2.1. Case $1 \leq p<\infty$. The following result provides an explicit characterization of the dual of Henig cones $\ell_{+, \varepsilon}^{p}$. We use the characterization of the dual of dilating cone given in [15, Theorems 4.1] (see also [15, Theorem 4.2]). As in the previous case, this result is originally given for a Hilbert space, but its proof only uses general Banach space properties and can be easily extended to any Banach space.

Proposition 2.2. For $p \in[1, \infty)$, the dual of the Henig dilating cone $\ell_{+, \varepsilon}^{p}$ is given by

$$
\left(\ell_{+, \varepsilon}^{p}\right)^{*}=\left\{\lambda=\left\{\lambda_{i}\right\}_{i \in \mathbb{N}} \in \ell_{+}^{q}: \lambda_{i} \geq \beta_{i}^{p} \varepsilon\|\lambda\|_{q}, \text { for every } i \in \mathbb{N}\right\} .
$$


Proof. Let $\lambda \in\left\{\lambda=\left\{\lambda_{i}\right\}_{i \in \mathbb{N}} \in \ell_{+}^{q}: \lambda_{i} \geq \beta_{i}^{p} \varepsilon\|\lambda\|_{q}\right\}$ be arbitrary. Then, for every

$$
\boldsymbol{\theta}=\left\{\theta_{i}\right\}_{i \in \mathbb{N}} \in \Theta_{p},
$$

we have

$$
\lambda(\boldsymbol{\theta})=\sum_{i=1}^{\infty} \lambda_{i} \theta_{i} \geq \sum_{i=1}^{\infty} \theta_{i} \beta_{i}^{p} \varepsilon\|\lambda\|_{q}=\varepsilon\|\lambda\|_{q}\left(\sum_{i=1}^{\infty} \theta_{i} \beta_{i}^{p}\right)=\varepsilon\|\lambda\|_{q} \beta_{p}(\boldsymbol{\theta})=\varepsilon\|\lambda\|_{q},
$$

and by applying [15, Theorem 4.1], we get $\lambda \in\left(\ell_{+, \varepsilon}^{p}\right)^{*}$.

For the converse, assume that there is $\mu \in\left(\ell_{+, \varepsilon}^{p}\right)^{*}$ such that for some $i_{0} \in \mathbb{N}$, we have

$$
\mu_{i_{0}}<\beta_{i_{0}}^{p} \varepsilon\|\mu\|_{q} .
$$

By defining

$$
\overline{\boldsymbol{\theta}}:=\frac{\mathbf{e}_{i_{0}}}{\beta_{p}\left(\mathbf{e}_{i_{0}}\right)}=\frac{\mathbf{e}_{i_{0}}}{\beta_{i_{0}}^{p}} \in \Theta_{p},
$$

we obtain from the above inequality that

$$
\mu(\overline{\boldsymbol{\theta}})=\mu\left(\frac{\mathbf{e}_{i_{0}}}{\beta_{i_{0}}^{p}}\right)=\frac{\mu_{i_{0}}}{\beta_{i_{0}}^{p}}<\varepsilon\|\mu\|_{q},
$$

which contradicts [15, Theorem 4.1], since we have $\mu \in\left(\ell_{+, \varepsilon}^{p}\right)^{*}$. The proof is complete.

As a consequence of Proposition 2.2, the Henig conical regularization scheme given in Proposition 2.1 has the following explicit (sequential) form for the $\ell^{p}$-constrained case.

Proposition 2.3. For each $\varepsilon \in(0,1)$, there is a unique solution $x_{p}^{\varepsilon}$ to problem $\left(Q_{p}^{\varepsilon}\right)$. Moreover, the regularized solutions $\left\{x_{p}^{\varepsilon}\right\}$ converge strongly to $\bar{x}_{p}$, as $\varepsilon \rightarrow 0$. Finally, for each $\varepsilon \in(0,1)$, there exists a Lagrange multiplier $\mu_{p}^{\varepsilon}=\left\{\mu_{i}^{p, \varepsilon}\right\}_{i \in \mathbb{N}} \in \ell_{+}^{q}$ such that

$$
\begin{aligned}
\varepsilon \beta_{i}^{p}\left\|\mu_{p}^{\varepsilon}\right\|_{q} & \leq \mu_{i}^{p, \varepsilon}, \\
D J\left(x_{p}^{\varepsilon}\right)+\sum_{i=1}^{\infty} \mu_{i}^{p, \varepsilon} G_{i}^{p} & =0, \\
\mu_{i}^{p, \varepsilon}\left(\left(G x_{p}^{\varepsilon}\right)_{i}-\mathbf{w}_{i}\right) & =0, \\
\left(G x_{p}^{\varepsilon}\right)_{i}-\mathbf{w}_{i} & \leq 0 \text { for every } i \in \mathbb{N},
\end{aligned}
$$

where we follow the notation $\mathbf{G} \mathbf{x}_{\mathbf{p}}^{\varepsilon}=\left\{\left(G x_{p}^{\varepsilon}\right)_{i}\right\}_{i \in \mathbb{N}}$, and $G_{i}: X \rightarrow \mathbb{R}$ are the projection maps defined by $G_{i}=e_{i}^{*} \circ G$ for every $i \in \mathbb{N}$.

In the following, by $\mu_{p}^{\varepsilon}$, we denote any multiplier satisfying conditions (2.2). Without any loss of generality, we assume $\left\{\mu_{p}^{\varepsilon}\right\} \neq 0$, otherwise we get the trivial case $x_{p}^{\varepsilon}=\bar{x}_{p}$. More precisely, if $\mu_{p}^{\varepsilon}=0$, then by KKT condition (2.2a), we obtain $D J\left(x_{p}^{\varepsilon}\right)=0$. Since $x_{p}^{\varepsilon} \rightarrow \bar{x}_{p}$, by continuity, we have $D J\left(\bar{x}_{p}\right)=0$. Because $\bar{x}_{p} \in F_{p}^{\varepsilon}$ is a feasible point of problem $\left(Q_{p}^{\varepsilon}\right), \bar{x}_{p}$ verifies KKT conditions (2.2) with multiplier $\bar{\mu}_{p}=\mu_{p}^{\varepsilon}=0$. By convexity of the problem, KKT are sufficient optimality conditions and consequently $\bar{x}_{p}=x_{p}^{\varepsilon}$.

Error estimates for the regularization error were established in [12, Theorem 3.2] for the general case. In our case, the result reads as follows. 
Proposition 2.4. Let $\delta_{p}^{\varepsilon}:=\varepsilon\left\|\mu_{p}^{\varepsilon}\right\|_{q}$. Then, $\left\{\delta_{p}^{\varepsilon}\right\} \rightarrow 0$. Moreover, the following estimate holds as $\varepsilon \rightarrow 0$ :

$$
\frac{\kappa}{2}\left\|\bar{x}_{p}-x_{p}^{\varepsilon}\right\|_{X}^{2}+\frac{1}{2}\left\|S \bar{x}_{p}-S x_{p}^{\varepsilon}\right\|_{V}^{2} \leq J\left(\bar{x}_{p}\right)-J\left(x_{p}^{\varepsilon}\right)-\delta_{p}^{\varepsilon}\left|\beta_{p}\left(\overline{\mathbf{q}}_{p}\right)\right| .
$$

If $\left(Q_{p}\right)$ is regular, then we can establish a priori error estimates in terms of the parameter. Recall that $\left(Q_{p}\right)$ is regular, if there exists a Lagrange multiplier $\bar{\mu}_{p} \in\left(\ell_{+}^{p}\right)^{*}$ such that

$$
\begin{aligned}
D J\left(\bar{x}_{p}\right)+\bar{\mu}_{p} \circ G & =0, \\
\bar{\mu}_{\mathbf{p}}\left(\mathbf{G} \overline{\mathbf{x}}_{\mathbf{p}}-\mathbf{w}\right) & =0, \\
\mathbf{G} \overline{\mathbf{x}}_{\mathbf{p}}-\mathbf{w} & \leq \leq_{\ell_{+}^{p}} 0 .
\end{aligned}
$$

In general, applying [12, Theorem 3.3], a priori estimate for the regularization error in terms of parameter $\varepsilon$ can be established. For the sake of completeness we give the result for our particular case.

Proposition 2.5. Assume that problem $\left(Q_{p}\right)$ is regular. Then the norm of the regularized multipliers $\left\{\left\|\mu_{p}^{\varepsilon}\right\|_{q}\right\}$ remain bounded for sufficiently small $\varepsilon$. Furthermore, there exists a constant $c>0$, independent of $\varepsilon$, such that

$$
\frac{\kappa}{2}\left\|\bar{x}_{p}-x_{p}^{\varepsilon}\right\|_{X}^{2}+\frac{1}{2}\left\|S \bar{x}_{p}-S x_{p}^{\varepsilon}\right\|_{V}^{2} \leq c \varepsilon .
$$

If the constraint space is a reflexive Banach space, the norm boundedness of the regularized multipliers is equivalent to the regularity of the original problem, see [15, Corollary 3.5]. In this case, since $\ell^{p}$ is reflexive for $p \in(1, \infty)$, we have the corresponding result .

Corollary 2.6. Assume $1<p<\infty$. Problem $\left(Q_{p}\right)$ is regular, if and only if, $\left\{\left\|\mu_{p}^{\varepsilon}\right\|_{q}\right\}$ is bounded for $\varepsilon$ small enough.

2.2. Case $p=\infty$. In this case, $\operatorname{int}\left(\ell_{+}^{\infty}\right) \neq \emptyset$, and Slater constraint qualification (1.1) is easy to verify. The following result follows from analogous arguments used above.

Proposition 2.7. For $\varepsilon \rightarrow 0$, we have

$$
\frac{\kappa}{2}\left\|\bar{x}_{\infty}-x_{\infty}^{\varepsilon}\right\|_{X}^{2}+\frac{1}{2}\left\|S \bar{x}_{\infty}-S x_{\infty}^{\varepsilon}\right\|_{V}^{2} \leq J\left(x_{\infty}^{\varepsilon}\right)-J\left(\bar{x}_{\infty}\right) .
$$

Furthermore, if $\left(Q^{\infty}\right)$ is regular, in particular, if Slater constraint qualification (1.1) holds, then there is a constant $c>0$, independent of $\varepsilon$, such that for $\varepsilon \rightarrow 0$, we have

$$
\frac{\kappa}{2}\left\|\bar{x}_{\infty}-x_{\infty}^{\varepsilon}\right\|_{X}^{2}+\frac{1}{2}\left\|S \bar{x}_{\infty}-S x_{\infty}^{\varepsilon}\right\|_{V}^{2} \leq c \varepsilon .
$$

By combining the previous results, we can establish the main result of the section. If the solution of problem $\left(Q^{\infty}\right)$ enjoys more regularity, more precisely if $\bar{x}_{\infty} \in F_{\bar{p}}$ for some $\bar{p}<\infty$, then we can approximate $\bar{x}_{\infty}$ by a sequence of regularized solutions $\left\{x_{\bar{p}}^{\varepsilon}\right\}$ associated with a (nonsingular) $\ell^{\bar{q}}$ multiplier, where $\bar{q}$ denotes the conjugate exponent of $\bar{p}$.

Theorem 2.8. Assume that $\mathbf{w} \in \ell^{\bar{p}}, \bar{x}_{\infty} \in F_{\bar{p}}$ for some $\bar{p} \in[1, \infty)$. Then $\bar{x}_{\infty}=\bar{x}_{\bar{p}}$ can be approximated by regularized solutions $\left\{x_{\bar{p}}^{\varepsilon}\right\}$ verifying (2.2) and such that for $\varepsilon \rightarrow 0$,

$$
\frac{\kappa}{2}\left\|\bar{x}_{\infty}-x_{\bar{p}}^{\varepsilon}\right\|_{X}^{2}+\frac{1}{2}\left\|S \bar{x}_{\infty}-S x_{\bar{p}}^{\varepsilon}\right\|_{V}^{2} \leq J\left(\bar{x}_{\infty}\right)-J\left(x_{\bar{p}}^{\varepsilon}\right)-\delta_{\bar{p}}^{\varepsilon}\left|\beta_{p}\left(\overline{\mathbf{q}}_{\bar{p}}\right)\right| .
$$

where we recall $\delta_{\bar{p}}^{\varepsilon}=\varepsilon\left\|\mu_{\bar{p}}^{\mathcal{E}}\right\|_{\bar{q}}$. 
Proof. The containment $F_{\bar{p}, 0} \subset F_{\infty, 0}$ implies $J\left(\bar{x}_{\infty}\right) \leq J\left(\bar{x}_{\bar{p}}\right)$. By hypothesis $\bar{x}_{\infty} \in F_{\bar{p}, 0}$, therefore by uniqueness $\bar{x}_{\infty}=\bar{x}_{\bar{p}}$. The remaining part of the proof follows by applying Proposition 2.1 and Proposition 2.4 .

\section{A Linear Convergence Rate For the Regularization ERror}

For a fixed $p \in[1, \infty]$, our aim here is to establish a linear convergence rate

$$
\left\|x_{p}^{\varepsilon}-\bar{x}_{p}\right\|_{X}=\mathscr{O}(\varepsilon)
$$

We define

$$
\mathbb{T}:=\left\{d \in X: \exists\left\{\varepsilon_{n}\right\} \downarrow 0 \text { such that }\left\{\frac{x_{p}^{\varepsilon_{n}}-\bar{x}_{p}}{\left\|x_{p}^{\varepsilon_{n}}-\bar{x}_{p}\right\|_{X}}\right\} \rightarrow d \text { as } n \rightarrow \infty\right\} .
$$

Since $X$ is reflexive, we have $\mathbb{T} \neq \emptyset$. In the following, ker denotes the kernel of a linear map.

The following results are inspired by the research conducted in [19], where we exclude the pathological case $x_{p}^{\varepsilon} \neq \bar{x}_{p}$.

Proposition 3.1. Let $\left(Q_{p}\right)$ be regular, and let $\bar{\mu}_{p}$ any multiplier verifying KKT condition (2.6) and $x_{p}^{\varepsilon} \neq \bar{x}_{p}$, for $\varepsilon$ sufficiently small. Then the following statements are equivalent:

(i): There exists a constant $c>0$ such that

$$
\left|D J\left(\bar{x}_{p}\right)(d)\right| \geq c, \text { for every } d \in \mathbb{T} .
$$

(ii): There exists a constant $c>0$, independent of $\varepsilon$, such that

$$
c\left\|x_{p}^{\varepsilon}-\bar{x}_{p}\right\|_{X} \leq \bar{\mu}_{p}\left(\mathbf{q}_{p}^{\varepsilon}\right), \text { for } \varepsilon \text { small enough. }
$$

(iii): $\operatorname{ker}\left(\bar{\mu}_{p} \circ G\right) \cap \mathbb{T}=\emptyset$.

(iv): $\operatorname{ker} D J\left(\bar{x}_{p}\right) \cap \mathbb{T}=\emptyset$.

Proof. (i) $\Leftrightarrow$ (ii): This equivalence follows by using basically the same reasoning as in [19, Proposition 3.2].

(iii) $\Leftrightarrow($ iv): It is a direct consequence of KKT condition (2.6a).

(i) $\Rightarrow$ (iv): It is immediate.

(iv) $\Rightarrow$ (i): Suppose that (i) does not hold. Therefore, we can take a sequence $\left\{d_{n}\right\} \subset \mathbb{T}$ such that $D J\left(\bar{x}_{p}\right)\left(d_{n}\right) \rightarrow 0$. Since every norm is weakly lower semicontinuous, every element of the set $\mathbb{T}$ is norm bounded by 1 . By the reflexivity of $X$, renaming subsequences if necessary, we can assume that $d_{n} \rightarrow d_{0}$ as $n \rightarrow \infty$ for some $d_{0} \in \mathbb{T}$, which belongs to $\mathbb{T}$ since $\mathbb{T}$ is sequentially weakly closed by definition. By continuity, from $D J\left(\bar{x}_{p}\right)\left(d_{n}\right) \rightarrow 0$, we have $D J\left(\bar{x}_{p}\right)\left(d_{0}\right)=0$ which contradicts (iv), and the proof is complete.

Motivated by Proposition 3.1, we formulate the following assumption:

$$
\operatorname{ker} D J\left(\bar{x}_{p}\right) \cap \mathbb{T}=\emptyset .
$$

Theorem 3.2. Assume that problem $\left(Q_{p}\right)$ is regular and (3.1) holds. Then, there exists a constant $c$, independent of $\varepsilon$, such that

$$
\left\|\bar{x}_{p}-x_{p}^{\varepsilon}\right\|_{X} \leq c \varepsilon
$$


Proof. We will consider $\bar{x}_{p} \neq x_{p}^{\varepsilon}$, on the contrary, (3.2) is trivially verified. By hypothesis, problem is regular and condition (3.1) is verified. Applying Proposition 3.1, we have

$$
k\left\|x_{p}^{\varepsilon}-\bar{x}_{p}\right\|_{X} \leq \bar{\mu}_{p}\left(\mathbf{q}_{p}^{\varepsilon}\right)
$$

for a constant $k>0$ and $\varepsilon$ small enough. On other hand, following the proof of Theorem 2.8, we can prove $\bar{\mu}_{p}\left(\mathbf{q}_{p}^{\varepsilon}\right) \leq c \varepsilon$, thus renaming constant (3.3), we get $\left\|x_{p}^{\varepsilon}-\bar{x}_{p}\right\|_{X} \leq c \varepsilon$.

For a Hilbert constraint space case, in [19] it is shown that $\left\|\bar{x}_{\infty}-x_{\infty}^{\varepsilon}\right\|_{X}=\mathscr{O}(\varepsilon)$ implies the regularity of the problem. This result remains valid in reflexive Banach spaces as well. As a consequence of this fact, and Theorem 2.5 and Theorem 3.2, we have the following:

Corollary 3.3. If $p \in(1, \infty)$ and (3.1) holds, then the following are equivalent:

(i): $\left(Q_{p}\right)$ is regular.

(ii): $\left\|\bar{x}_{p}-x_{p}^{\varepsilon}\right\|_{X}=\mathscr{O}(\varepsilon)$ as $\varepsilon \rightarrow 0$.

(iii): $\left\{\left\|\mu_{p}^{\varepsilon}\right\|_{q}\right\}$ is bounded for $\varepsilon$ small enough.

\section{NUMERICAL EXAMPLES}

To illustrate the developed theory, we now consider two examples which are quadratic modifications of the linear examples presented in [22].

Example 1. Non-existence of the multipliers in $\ell^{p}$. We consider the quadratic optimization problem

$$
\text { Minimize } J(x)=\frac{1}{2}(x-1)^{2} \quad \text { subject to } G x \leq_{\ell_{+}^{p}} \mathbf{w}, \quad x \in \mathbb{R},
$$

where $G: \mathbb{R} \rightarrow \ell^{p}$ is the bounded linear operator defined by $G x=x \mathbf{a}=\left\{\frac{x}{i^{2}}\right\}_{i \in \mathbb{N}}$, with $\mathbf{a}=\left\{\frac{1}{i^{2}}\right\}_{i \in \mathbb{N}}$, and $\mathbf{w} \in \ell^{p}$ is the sequence defined by $\mathbf{w}=\left\{\frac{1}{i^{3}}\right\}_{i \in \mathbb{N}}$. This problem is of type $\left(Q_{p}\right)$ by taking $X=\mathbb{R}$, $S=v_{d}=0, \kappa=1, x_{d}=1$. For $p \in[1, \infty]$, the feasible set reads:

$$
F_{p}=\left\{x \in \mathbb{R}: G x-\mathbf{w} \in-\ell_{+}^{p}\right\}=\left\{x \in \mathbb{R}: \frac{x}{i^{2}} \leq \frac{1}{i^{3}} \text { for every } i=1, \ldots, \infty\right\}=(-\infty, 0] .
$$

Clearly $\bar{x}_{p}=0$ is the unique solution to $\left(Q_{p}\right)$.

Problem $\left(Q_{p}\right)$ has no Lagrange multiplier for $p<\infty$. Otherwise, there would be $\bar{\mu}_{p} \in\left(\ell_{+}^{p}\right)^{*} \equiv \ell_{+}^{q}$ such that

$$
D J\left(\bar{x}_{p}\right)+\bar{\mu}_{p} \circ G=0 \Leftrightarrow-1+\bar{\mu}_{p} \circ G=0 \Leftrightarrow \bar{\mu}_{p} \circ G=1,
$$

and

$$
\bar{\mu}_{p}\left(G \bar{x}_{p}-\mathbf{w}\right)=0 \Leftrightarrow \bar{\mu}_{p}(\mathbf{w})=0 \Leftrightarrow \sum_{i=1}^{\infty} \bar{\mu}_{i}^{p} w_{i}=0 \Leftrightarrow \sum_{i=1}^{\infty} \bar{\mu}_{i}^{p} i^{-3}=0 .
$$

From this last expression, since $\bar{\mu}_{i}^{p} \geq 0$, we have $\bar{\mu}_{i}^{p}=0$. Therefore $\bar{\mu}_{p}=0$, and from (4.1), we get $\bar{\mu}_{p} \circ G=0=1$ which is impossible.

For the case $p=\infty$, this reasoning also proves that the Lagrange multiplier, if it exists, would be necessarily a purely additive multiplier. Moreover Slater constraint qualification does not apply, whereas the numerical results suggest problem $\left(Q_{\infty}\right)$ has not dual solution.

We now consider the Henig conically regularized problems:

$$
\text { Minimize } J(x)=\frac{1}{2}(x-1)^{2} \quad \text { subject to } G x \leq_{\ell_{+, \varepsilon}^{p}} \mathbf{w}, \quad x \in \mathbb{R} .
$$


Note that condition (2.1) is verified, since $\overline{\mathbf{q}}_{p}=-\mathbf{w} \neq \mathbf{0}$. Following Theorem 2.1, this problem is regular and we can solve the KKT conditions. In our numerical computations, we will use the Lagrange dual problem which can be computed explicitly. The associated Lagrangian is then given by

$$
L\left(x, \mu^{\varepsilon}\right)=\frac{1}{2}(x-1)^{2}+\mu^{\varepsilon}(G x-\mathbf{w})=\frac{1}{2}(x-1)^{2}+\mu^{\varepsilon}(\mathbf{a}) x-\mu^{\varepsilon}(\mathbf{w}),
$$

while the corresponding Lagrangian dual function is given by

$$
g\left(\mu^{\varepsilon}\right)=\max _{x \in \mathbb{R}} L\left(x, \mu^{\varepsilon *}\right)=L\left(1-\mu^{\varepsilon}(\mathbf{a}), \mu^{\varepsilon *}\right)=\mu^{\varepsilon}(\mathbf{a})-\frac{1}{2} \mu^{\varepsilon *}(\mathbf{a})^{2}-\mu^{\varepsilon}(\mathbf{w})
$$

for every $\mu^{\varepsilon} \in\left(\ell_{+, \varepsilon}^{p}\right)^{*}$. Now, applying the characterization of $\left(\ell_{+, \varepsilon}^{p}\right)^{*}$ given in Proposition 2.2, the dual Lagrange problem can be written in the following way:

$$
\begin{aligned}
& \text { Maximize } g\left(\mu^{\varepsilon}\right)=\sum_{i=1}^{\infty} \frac{\mu_{i}^{\varepsilon}}{i^{2}}-\frac{1}{2}\left(\sum_{i=1}^{\infty} \frac{\mu_{i}^{\varepsilon}}{i^{2}}\right)^{2}-\sum_{i=1}^{\infty} \frac{\mu_{i}^{\varepsilon}}{i^{3}} \\
& \text { subject to } \mu_{i}^{\varepsilon} \geq \beta_{i}^{p} \varepsilon\|\mu\|_{q} \text { for every } i \in \mathbb{N}, \mu=\left\{\mu_{i}^{\varepsilon}\right\}_{i \in \mathbb{N}} \in \ell^{q} .
\end{aligned}
$$

In this case, strong duality holds, $\left(D_{p}^{\varepsilon}\right)$ is solvable and any solution $\mu^{\varepsilon}=\left\{\mu_{i}^{\varepsilon}\right\}$ is a regular multiplier of $\left(Q_{p}^{\varepsilon}\right)$. Furthermore, the optimal values of $\left(Q_{p}^{\varepsilon}\right)$ and $\left(D_{p}^{\varepsilon}\right)$ coincide. In order to compute numerically the solution to problem $\left(D_{p}^{\varepsilon}\right)$, we truncate every sequence by considering the first $n=1000$ elements. In Table 1 we present numerical results for three exponents $p \in\{1,2,50\}$ and a finite sequence of parameter $\varepsilon$ for each case. In our computation we consider $\beta=\left\{i^{-2}\right\}_{i \in \mathbb{N}} \in \ell^{1}$.

We have solved numerically $\left(D_{p}^{\varepsilon}\right)$ by using CVX (see [6]). Numerical results show some slightly differences between the selected exponents, with fast convergence results for smaller $p$. This behaviour persists for larger exponents. Taking into account that for smaller $\varepsilon$ the error rate convergence stagnates, since truncation error dominates, experimentally one can compute a convergence rate $\mathscr{O}\left(\varepsilon^{k}\right)$ with exponent $k$ varying between 0.3 and 0.4 . This rate is below $\mathscr{O}(\sqrt{\varepsilon})$, which agrees with the fact that problem is not regular. Furthermore, one also notes the unbounded multipliers which agrees with the fact that problem is not regular, specially in the case of reflexive exponents $p \in\{2,50\}$ where Corollary 2.6 holds.

Finally, let us emphasize that Theorem 2.8 can be applied for every index $\bar{p}<\infty$, and it is an example of a problem with non-regular multiplier in $\ell^{\infty}$ which can be approximated by regularized solutions associated with $\ell^{p}$ multipliers.

Example 2. Singular multipliers. We consider the $\ell^{\infty}$-constrained problem

$$
\text { Minimize } J(x)=\frac{1}{2}(x-1)^{2} \quad \text { subject to } G x \leq_{\ell_{+}^{\infty}} \mathbf{w}, \quad x \in \mathbb{R},
$$

where $G: \mathbb{R} \rightarrow \ell^{\infty}$ is the bounded linear operator defined by $G x=\{x\}_{i \in \mathbb{N}}=x \mathbf{1}$, where $\mathbf{1}=\{1\}_{i \in \mathbb{N}} \in \ell^{\infty}$, and $\mathbf{w} \in \ell^{p}$ is the sequence defined by $\mathbf{w}:=\left\{\frac{1}{i}\right\}_{i \in \mathbb{N}}$. Reasoning as before,

$$
x \in F_{\infty} \Leftrightarrow G x-\mathbf{w} \in-\ell_{+}^{\infty} \Leftrightarrow x \leq \frac{1}{i} \text { for every } i \in \mathbb{N} \Leftrightarrow x \leq 0,
$$

thus $F_{\infty}=(-\infty, 0]$. Clearly $\bar{x}_{\infty}=0$ is the unique solution to $\left(Q_{\infty}\right)$. On the other hand, Slater constraint qualification (1.1) is verified. For example, taking $\tilde{x}=-1$

$$
G \tilde{x}-\mathbf{w}=\left\{-1-\frac{1}{i}\right\}_{i \in \mathbb{N}} \leq_{\ell_{+}^{\infty}}\{-1\}_{i \in \mathbb{N}} \in-\text { int } \ell_{+}^{\infty} .
$$




\begin{tabular}{|c|c|c|c|c|c|c|}
\hline$\varepsilon$ & $E(\varepsilon)=\left|x_{1}^{\varepsilon}\right|$ & $\left\|\mu_{1}^{\varepsilon}\right\|_{\infty}$ & $E(\varepsilon)=\left|x_{2}^{\varepsilon}\right|$ & $\left\|\mu_{2}^{\varepsilon}\right\|_{2}$ & $E(\varepsilon)=\left|x_{50}^{\varepsilon}\right|$ & $\left\|\mu_{50}^{\varepsilon}\right\|_{\frac{50}{49}}$ \\
\hline $1 . \mathrm{e}-01$ & $3.602 \mathrm{e}-01$ & 1.27 & $5.602 \mathrm{e}-01$ & 1.412 & $5.852 \mathrm{e}-01$ & 2.460 \\
\hline $1 . \mathrm{e}-02$ & $1.344 \mathrm{e}-01$ & 6.055 & $2.734 \mathrm{e}-01$ & 9.332 & $3.109 \mathrm{e}-01$ & $1.485 \mathrm{e} 01$ \\
\hline $1 . \mathrm{e}-03$ & $4.556 \mathrm{e}-02$ & $2.052 \mathrm{e} 01$ & $1.180 \mathrm{e}-01$ & $4.485 \mathrm{e} 02$ & $1.542 \mathrm{e}-01$ & $7.522 \mathrm{e} 01$ \\
\hline $1 . \mathrm{e}-04$ & $1.550 \mathrm{e}-02$ & $6.718 \mathrm{e} 01$ & $4.851 \mathrm{e}-02$ & $1.908 \mathrm{e} 02$ & $7.350 \mathrm{e}-02$ & $3.697 \mathrm{e} 02$ \\
\hline $1 . \mathrm{e}-05$ & $5.960 \mathrm{e}-03$ & $2.105 \mathrm{e} 02$ & $1.957 \mathrm{e}-02$ & $7.796 \mathrm{e} 02$ & $3.452 \mathrm{e}-02$ & $1.746 \mathrm{e} 03$ \\
\hline $1 . \mathrm{e}-06$ & $3.084 \mathrm{e}-03$ & $5.021 \mathrm{e} 03$ & $7.925 \mathrm{e}-03$ & $3.063 \mathrm{e} 03$ & $2.411 \mathrm{e}-02$ & $6.473 \mathrm{e} 03$ \\
\hline $1 . \mathrm{e}-07$ & $2.380 \mathrm{e}-03$ & $5.601 \mathrm{e} 03$ & $3.833 \mathrm{e}-03$ & $7.508 \mathrm{e} 03$ & $1.823 \mathrm{e}-02$ & $3.124 \mathrm{e} 04$ \\
\hline $1 . \mathrm{e}-08$ & $2.215 \mathrm{e}-03$ & $5.770 \mathrm{e} 03$ & $2.963 \mathrm{e}-03$ & $8.582 \mathrm{e} 03$ & $1.097 \mathrm{e}-02$ & $4.921 \mathrm{e} 04$ \\
\hline
\end{tabular}

TABLE 1. Example 1. Problems $\left(Q^{1}\right),\left(Q^{2}\right)$, and $\left(Q^{50}\right)$

Therefore, KKT conditions (2.6) are solvable and there exists $\bar{\mu}_{\infty} \in\left(\ell_{+}^{\infty}\right)^{*}$ such that

$$
D J\left(\bar{x}_{\infty}\right)+\bar{\mu}_{\infty} \circ G=0 \Leftrightarrow \bar{\mu}_{\infty} \circ G=1,
$$

and

$$
\bar{\mu}_{\infty}\left(G \bar{x}_{\infty}-\mathbf{w}\right)=0 \Leftrightarrow \bar{\mu}_{\infty}(\mathbf{w})=0 \Leftrightarrow \sum_{i=1}^{\infty} \bar{\mu}_{i}^{\infty} w_{i}+\bar{\mu}_{\text {sing }}(\mathbf{w})=0 .
$$

By [1, Lemma 16.30], we have $\bar{\mu}_{\text {sing }}(\mathbf{w})=0$, consequently (4.3) is equivalent to $\sum_{i=1}^{\infty} \bar{\mu}_{i}^{\infty} i^{-1}=0$. From this last expression, since $\mu_{i}^{\infty} \geq 0$, we necessarilly have that $\mu_{i}^{\infty}=0$. Therefore $\bar{\mu}_{\infty}=\bar{\mu}_{\text {sing }}$ is a purely singular additive multiplier. Furthermore from (4.2)

$$
\left(\bar{\mu}_{\infty} \circ G\right)(1)=1 \Leftrightarrow \bar{\mu}_{\text {sing }}(\mathbf{1})=1 .
$$

We solve numerically this problem by using the dual problem. The Lagrangian reads

$$
L\left(x, \mu^{\varepsilon}\right)=\frac{1}{2}(x-1)^{2}+\mu^{\varepsilon}(G x-\mathbf{w})=\frac{1}{2}(x-1)^{2}+\mu^{\varepsilon}(\mathbf{1}) x-\mu^{\varepsilon}(\mathbf{w}),
$$

and the corresponding Lagrangian dual function is given by

$$
g\left(\mu^{\varepsilon}\right)=\max _{u \in \mathbb{R}} L\left(u, \mu^{\varepsilon}\right)=L\left(1-\mu^{\varepsilon}(\mathbf{1}), \mu^{\varepsilon}(\mathbf{1})\right)=\mu^{\varepsilon}(\mathbf{1})-\frac{1}{2} \mu^{\varepsilon}(\mathbf{1})^{2}-\mu^{\varepsilon}(\mathbf{w}),
$$

for every $\mu^{\varepsilon} \in\left(\ell_{+, \varepsilon}^{\infty}\right)^{*}$. The dual Lagrange problem can be written in the following way

$$
\text { Maximize } g\left(\mu^{\varepsilon *}\right)=\mu^{\varepsilon}(\mathbf{1})-\frac{1}{2} \mu^{\varepsilon}(\mathbf{1})^{2}-\mu^{\varepsilon}(\mathbf{w}) \text { subject to } \mu^{\varepsilon} \in\left(\ell_{+, \varepsilon}^{\infty}\right)^{*} . \quad\left(D_{\infty}^{\varepsilon}\right)
$$

In this case, we do not have the characterization of the dual elements given in Proposition 2.2, but we can use similar ideas to approximate this cone. Again we consider $\beta=\left\{\frac{1}{i^{2}}\right\}_{i \in \mathbb{N}}$, in this case $\|\beta\|_{\infty}=1$, thus $\beta_{\infty}=\beta$. By definition, for every $\mu \in\left(\ell_{+}^{\infty}\right)^{*}$, we have

$$
\|\mu\|_{\left(\ell_{+}^{\infty}\right)^{*}}=\mu(\mathbf{1})=\sum_{i=1}^{n} \mu_{i}+\mu_{\text {sing }}(\mathbf{1}) .
$$

By taking $\theta=\frac{\mathbf{e}_{i}}{\beta\left(\mathbf{e}_{i}\right)}=\frac{\mathbf{e}_{i}}{\beta_{i}} \in \Theta_{\infty}$, for an arbitrary $\mu \in\left(\ell_{+}^{\infty}\right)^{*}$, we have

$$
\mu\left(\frac{\mathbf{e}_{i}}{\beta\left(\mathbf{e}_{i}\right)}\right)=\frac{\mu_{i}}{\beta_{i}} \geq \varepsilon\|\mu\|_{\left(\ell_{+}^{\infty}\right)^{*}} \Leftrightarrow \mu_{i} \geq \varepsilon \beta_{i} \mu(\mathbf{1})
$$


equivalently

$$
\mu_{i} \geq \varepsilon\left(\sum_{i=1}^{\infty} \mu_{i}+\mu_{\text {sing }}(\mathbf{1})\right)=\varepsilon\left(\|\mu\|_{1}+\mu_{\text {sing }}(\mathbf{1})\right),
$$

for every $i \in \mathbb{N}$. Therefore

$$
\left(\ell_{+, \varepsilon}^{\infty}\right)^{*} \subset\left\{\mu \in\left(\ell_{+}^{\infty}\right)^{*}: \mu_{i} \geq \varepsilon \beta_{i}\left(\|\mu\|_{1}+\mu_{\text {sing }}(\mathbf{1})\right)\right\} .
$$

Based on (4.4), we solve numerically, the following relaxation of problem $\left(D_{\infty}^{\varepsilon}\right)$ :

$$
\begin{aligned}
& \text { Maximize } \sum_{i=1}^{\infty} \mu_{i}+\mu_{\text {sing }}(\mathbf{1})-\frac{1}{2}\left(\sum_{i=1}^{\infty} \mu_{i}+\mu_{\text {sing }}(\mathbf{1})\right)^{2}-\sum_{i=1}^{\infty} \mu_{i} \frac{1}{i} \\
& \text { subject to } \mu_{i} \geq \varepsilon \beta_{i}\left(\sum_{i=1}^{\infty} \mu_{i}+\mu_{\text {sing }}(\mathbf{1})\right) \forall i \in \mathbb{N}, \quad \sum_{i=1}^{\infty} \mu_{i}+\mu_{\text {sing }}(\mathbf{1}) \geq 0 .
\end{aligned}
$$

\begin{tabular}{|c|c|c|}
\hline$\varepsilon$ & $E(\varepsilon)=\left|x_{\infty}^{\varepsilon}\right|$ & $\left\|\mu_{\infty}^{\varepsilon}\right\|_{\left(\ell^{\infty}\right)^{*}}$ \\
\hline $1 . \mathrm{e}-01$ & $1.202 \mathrm{e}-01$ & $8.797 \mathrm{e}-01$ \\
\hline $1 . \mathrm{e}-02$ & $1.202 \mathrm{e}-02$ & $9.880 \mathrm{e}-01$ \\
\hline $1 . \mathrm{e}-03$ & $1.202 \mathrm{e}-03$ & $9.988 \mathrm{e}-01$ \\
\hline $1 . \mathrm{e}-04$ & $1.202 \mathrm{e}-04$ & 1 \\
\hline $1 . \mathrm{e}-05$ & $1.203 \mathrm{e}-05$ & 1 \\
\hline $1 . \mathrm{e}-06$ & $1.210 \mathrm{e}-06$ & 1 \\
\hline $1 . \mathrm{e}-07$ & $1.281 \mathrm{e}-07$ & 1 \\
\hline $1 . \mathrm{e}-08$ & $1.982 \mathrm{e}-08$ & 1 \\
\hline
\end{tabular}

TABLE 2. Example 2

We solve numerically this problem using the same approach as in previous example. Numerical results are presented in Table 2. In practice, the relaxation problem seems to work adequately as numerical results match the theorical results. Convergence rate exhibits a linear rate, $\left|x_{\infty}^{\varepsilon}-\bar{x}^{\varepsilon}\right|=\mathscr{O}(\varepsilon)$, which agrees with Theorem 3.2. In this sense, let us underline that hypothesis of Theorem 3.2 are verified. On the one hand problem $\left(Q_{\infty}\right)$ is regular, and on the other hand we have $\operatorname{ker} D J\left(\bar{x}_{\infty}\right)=\{0\}, \mathbb{T}=\{1\}$, therefore

$$
\operatorname{ker} D J\left(x^{\infty}\right) \cap \mathbb{T}=\emptyset
$$

is verified. Finally let us underline that for this problem $F_{p}=\emptyset$ for every $p<\infty$, henceforth we can not apply Theorem 2.8 .

\section{CONCLUSiOnS}

In this paper, we analyzed conical regularization to least-squares problems with $\ell_{+}^{p}$ linear constraints. For the case $p<\infty$, we showed the usefulness of the proposed scheme in dealing with the lack of Slatertype constraint qualification. On the other hand, for the case $p=\infty$, the developed framework aids with the existence of singular dual solutions. Besides providing an explicit construction for the regularization scheme, we also proved novel stability estimates concerning the perturbation error. Preliminary numerical results validate the theoretical results and suggest that these new ideas can be applied to many 
important mathematical models in economy and other related fields which can be formulated as a $\ell_{+}^{p}$ constrained model.

\section{Acknowledgments}

The second author was supported by National Science Foundation grant under Award No. 1720067. The first and the third authors were supported by Ministerio de Ciencia, Innovación y Universidades (MCIU), Agencia Estatal de Investigación (AEI) (Spain) and Fondo Europeo de Desarrollo Regional (FEDER) under project PGC2018-096899-B-I00 (MCIU/AEI/FEDER, UE).

\section{REFERENCES}

[1] C.D. Aliprantis, K.C. Border, Infinite dimensional analysis, A hitchhiker's guide, third edn. Springer, Berlin, 2006.

[2] A. Basu, K. Martin, C.T. Ryan, Strong duality and sensitivity analysis in semi-infinite linear programming, Math. Program. 161 (2017), 451-485.

[3] J.M. Borwein, D. Zhuang, Super efficiency in vector optimization, Trans. Amer. Math. Soc. 338 (1993), 105-122.

[4] A. Ghate, Circumventing the Slater conundrum in countably infinite linear programs, Eur. J. Oper. Res. 246 (2015), 708-720.

[5] A. Ghate, R.L. Smith, A linear programming approach to nonstationary infinite-horizon markov decision processes, Oper. Res. 61 (2013), 413-425.

[6] M. Grant, S. Boyd, CVX: Matlab software for disciplined convex programming, version 2.1. http://cvxr.com/cvx, 2014.

[7] R. Grinold, Convex infinite horizon programs, Math. Program. 25 (1983), 64-82.

[8] M. Henig, Proper efficiency with respect to cones, J. Optim. Theory Appl. 36 (1982), 387-407.

[9] M. Henig, A cone separation theorem, J. Optim. Theory Appl. 36 (1982), 451-455.

[10] L. Huerga, B. Jadamba, M. Sama, An extension of the Kaliszewski cone to non-polyhedral pointed cones in infinitedimensional spaces, J. Optim. Theory Appl. 181 (2019), 437-455.

[11] B. Jadamba, A.A. Khan, M. Sama, Regularization for state constrained optimal control problems by half spaces based decoupling, Systems \& Control Lett. 61 (2012), 707-713.

[12] B. Jadamba, A.A. Khan, M. Sama, Stable conical regularization by constructible dilating cones with an application to $\mathrm{L}^{p}$-constrained optimization problems, Taiwanese J. Math. 23 (2019), 1001-1023.

[13] B. Jadamba, A.A. Khan, R. López, M. Sama, Conical regularization for multi-objective optimization problems, J. Math. Anal. Appl. 479 (2019), 2056-2075.

[14] B. Jadamba, A.A. Khan, M. Sama, Error estimates for integral constraint regularization of state-constrained elliptic control problems, Comput. Optim. Appl. 67 (2017), 39-71.

[15] A.A. Khan, M. Sama, A new conical regularization for some optimization and optimal control problems: Convergence analysis and finite element discretization, Numeri. Funct. Anal. Optim. 34 (2013), 861-895.

[16] A.A. Khan, C. Z. Tammer, C. Zălinescu, Set-valued optimization, Springer, Heidelberg, 2015.

[17] P. Kogut, G. Leugering, R. Schiel, On Henig regularization of material design problems for quasi-linear p-biharmonic equation, Appl. Math. 7 (2016), 1547.

[18] P. I. Kogut, G. Leugering, R. Schiel, On the relaxation of state-constrained linear control problems via Henig dilating cones, Control \& Cybernetics 45 (2016), 131-162.

[19] R. López, M. Sama, Stability and sensivity analysis for conical regularization of linearly constrained least-squares problems in Hilbert spaces, J. Math. Anal. Appl. 456 (2017), 476-495.

[20] K. Martin, C.T. Ryan, M. Stern, The Slater conundrum: duality and pricing in infinite-dimensional optimization, SIAM J. Optim. 26 (2016), 111-138.

[21] A. Mas-Colell, W.R. Zame, Equilibrium theory in infinite dimensional spaces, Handbook of Mathematical Economics 4 (1991), 1835-1898.

[22] J. Ponstein, On the use of purely finitely additive multipliers in mathematical programming, J. Optim. Theory Appl. 33 (1981), 37-55. 
[23] R. Radner, Efficiency prices for infinite horizon production programmes, The Review of Economic Studies 34 (1967), 51-66.

[24] H.E. Romeijn, R.L. Smith, J.C. Bean, Duality in infinite dimensional linear programming, Math. Program. 53 (1992), 79-97.

[25] R. Schiel, Vector Optimization and Control with Partial Differential Equations and Pointwise State Constraints. Ph.D. thesis, FAU Erlangen-Nürnberg, (2014).

[26] T.C. Sharkey, H. E. Romeijn, A simplex algorithm for minimum-cost network-flow problems in infinite networks, Networks 52 (2008), 14-31. 